\title{
Predictors of cardiac allograft vasculopathy in pediatric heart transplant recipients
}

Kobayashi D, Du W, L'Ecuyer TJ. Predictors of cardiac allograft vasculopathy in pediatric heart transplant recipients.

Abstract: CAV remains a leading cause of late graft loss and mortality among survivors of pediatric heart transplantation. We sought to define the incidence of CAV and identify its predictors in pediatric heart transplant recipients. The OPTN/UNOS database was analyzed for pediatric recipients who underwent heart transplant between 1987 and 2011. The primary end-point is time from heart transplantation to development of CAV (CAV-free survival). To identify predictors of CAV-free survival, demographic and transplant data were analyzed by the Kaplan-Meier survival method and Cox proportional hazards regression. Of 5211 pediatric heart transplant recipients with at least one-yr follow-up, the incidence of CAV at five, 10 , and $15 \mathrm{yr}$ was $13 \%$, $25 \%$, and $54 \%$, respectively. Multivariate analysis found that risk of CAV was associated with the following variables: Recipient age 1-4 yr (HR 1.25), 5-9 yr (1.45), 10-18 yr (1.83), donor age $>18$ yr (1.34), re-transplantation (2.14), recipient black race (1.55), and donor cigarette use (1.54). Older recipient and donor age, recipient black race, donor cigarette use, and re-transplantation were highly associated with shorter CAV-free survival.

\section{Daisuke Kobayashi, Wei Du and Thomas J. L'Ecuyer}

Section of Pediatric Cardiology, Carman and Ann Adams Department of Pediatrics, Children's Hospital of Michigan, Wayne State University School of Medicine, Detroit, MI, USA

Key words: cardiac allograft vasculopathy orthotopic heart transplant - children - predictor

Daisuke Kobayashi, MD, Section of Pediatric Cardiology, Carman and Ann Adams Department of Pediatrics, Children's Hospital of Michigan, Wayne State University School of Medicine, 3901 Beaubien Blvd, Detroit, Ml 48201-2119, USA

Tel.: +1 3137455481

Fax: +1 3139930894

E-mail: dkobayas@dmc.org

Accepted for publication 16 April 2013
CAV remains a leading cause of graft loss and mortality among late survivors of heart transplantation (1). Despite improvements of post-transplant management in the current era, one-third of pediatric patients develop CAV within $10 \mathrm{yr}$ of transplant (2). Understanding the risk factors for CAV may lead to earlier diagnosis and better management, resulting in longer graft survival. However, data regarding risk factors for CAV in children remain scarce. The PHTS group recently reported CAV risk factors including older recipient and donor age and early rejection ( $\geq 2$ rejections within one yr of transplant) (3). In a report from the ISHLT, the only identified risk factor was recipient age $(\geq 11 \mathrm{yr})$ (2). Risk factors for the early development of CAV (within five yr of transplant) were re-transplant, PRA $>10 \%$, congenital heart disease, and

\footnotetext{
Abbreviations: CAV, cardiac allograft vasculopathy; DSA, donor-specific antibodies; HR, hazard ratio; ISHLT, International Society for Heart Lung Transplantation; OPTN, Organ Procurement and Transplantation Network; PHTS, Pediatric Heart Transplant Study; PRA, panel-reactive antibody; UNOS, United Network for Organ Sharing.
}

a steroid-free regimen at discharge for maintenance immunosuppression.

The OPTN is the unified transplant network operated by a private, non-profit organization to increase the effectiveness and efficiency of organ sharing and equity in the national system of organ allocation and increase the supply of donated organs available for transplantation. OPTN is administered by the UNOS, which is a private, non-profit organization that maintains a database containing organ transplant data for every transplant event that occurs in the United States (4). In our study, we sought to define the incidence of CAV and identify its predictors in pediatric heart transplant recipients, utilizing this database. Compared to previous studies, our work references the largest pediatric transplant data set in the United States. Furthermore, submission of data to the OPTN/UNOS database is mandatory. As a result, these data represent all programs within the United States. Although data in the OPTN/ UNOS database are not structured specifically to be a research database, this large data set with long patient follow-up enables us to evalu- 
ate the incidence and risk factors for CAV in pediatric heart transplant patients.

\section{Methods}

We performed a retrospective analysis of the OPTN/ UNOS database for pediatric patients aged $0-18 \mathrm{yr}$ who underwent heart transplant between October 1987 and December 2011 and had at least one-yr follow-up data. Patients who underwent heart and lung transplantation were excluded from this study. Patients who underwent heart re-transplantation were included. The primary endpoint is time from heart transplantation to development of CAV (CAV-free survival). Because the actual date of CAV diagnosis is not given in the OPTN/UNOS database, the date of the annual follow-up immediately following the CAV diagnosis is used instead. The CAV was not defined in the OPTN/UNOS database, but rather was defined by individual transplant centers and did not describe severity. Our study focus was to identify predictors of CAV using baseline demographic and immediate pre- and post-transplant data. Age mismatch was defined as donor-to-recipient age ratio $<0.8$ or $>2.0$. HLA and HLA DR mismatches were defined as $\geq 5$ loci and two loci mismatches, respectively. Significant renal insufficiency was defined as serum creatinine $>2 \mathrm{mg} / \mathrm{dL}$. In recipients, transfusion between listing and transplant was recorded. Recent and peak PRA refer to the most recent and peak value (percent) prior to transplant, respectively. Donor alcohol use was defined as a history of alcohol dependency. Donor cigarette use was defined as a history of smoking $>20$ pack yr. Early and late rejection was defined as acute rejection that occurred within and after one yr of transplant, respectively.

Statistical analysis was performed using SAS version 9.2 (Cary, NC, USA). Kaplan-Meier's product-limit method and log-rank test were first used to analyze CAV-free survival univariately to examine the association of all potential risk factors (including interaction items) with CAV. The risk factors that showed statistically significant association $(\mathrm{p}<0.05)$ with CAV-free survival were then included in multivariate analysis that utilized Cox proportional hazards regression along with backward model selection technique to arrive at our final predictive model. Interactions between risk factors for CAV were tested, and the few that were included in multivariate analysis were all dropped from the final model. Variables that had more than $50 \%$ missing values were excluded from our analysis. Missing data for those risk factors included in our final model for the prediction of CAV-free survival were examined, and the degree to which data was missing appeared to be random. No imputation for missing values was performed. A $p$ value $<0.05$ was considered statistically significant.

This study was approved by the Wayne State University Institutional Review Board. UNOS Standard Analysis and Research files provided de-identified data.

\section{Results}

\section{Demographics}

Among the 7914 pediatric heart transplant recipients registered in the database, 2703 patients who underwent heart-lung transplant or did not have one-yr follow-up were excluded (Table 1-3) .Of the 5211 heart transplant recipients analyzed
(Table 1), mean age was 6.5 yr with $44.1 \%$ female and $28.6 \%<1 \mathrm{yr}$ of age. The most common race was white $(61.5 \%)$, followed by black $(18.8 \%)$. The most common reason for heart transplant was cardiomyopathy $(48.3 \%)$, followed by congenital heart disease $(42.8 \%)$ and re-transplantation $(5.5 \%)$. Pretransplant morbidities in recipients included renal insufficiency $(2.6 \%)$ and diabetes $(0.4 \%)$. Recent and peak PRA $>10 \%$ prior to transplant were seen in $23.4 \%$ and $31.0 \%$ of recipients, respectively.

Table 2 shows the donor characteristics. The majority of donors was white $(62.9 \%)$ followed by black $(17.1 \%)$. Mean age of donors was $9.3 \mathrm{yr}$ with $42.3 \%$ female and $21.3 \%<1$ yr. Morbidities in donors included renal insufficiency $(4.8 \%)$, diabetes $(0.8 \%)$, hypertension $(1.3 \%)$, cigarette use $(4.5 \%)$, and alcohol use $(2.5 \%)$.

Transplant-related characteristics (Table 1) included the mean ischemic time of $3.5 \mathrm{~h}$ with $31.5 \%>4 \mathrm{~h}$. Early rejection was seen in $24.4 \%$ of recipients and late rejection in $17.6 \%$. Prior to discharge, a pacemaker was implanted in $1.0 \%$, and dialysis was required in $2.9 \%$ of the recipients.

Table 1. Recipient and transplant characteristics ( $N=5211$ )

\begin{tabular}{|c|c|c|}
\hline Group & Characteristics & Percent \\
\hline \multirow[t]{24}{*}{ Recipient } & \multicolumn{2}{|l|}{ Age (yr) } \\
\hline & $<1$ & 28.6 \\
\hline & $1-4$ & 20.4 \\
\hline & $5-9$ & 13.5 \\
\hline & $10-18$ & 37.5 \\
\hline & Age (mean \pm s.d.) & $6.5 \pm 6.2 \mathrm{yr}$ \\
\hline & \multicolumn{2}{|l|}{ Race } \\
\hline & Black & 18.8 \\
\hline & White & 61.5 \\
\hline & Others & 19.7 \\
\hline & Gender (female) & 44.1 \\
\hline & \multicolumn{2}{|l|}{ Indication for heart transplant } \\
\hline & Congenital heart disease & 42.8 \\
\hline & Cardiomyopathy & 48.3 \\
\hline & Re-transplant & 5.5 \\
\hline & Others & 3.4 \\
\hline & \multicolumn{2}{|l|}{ Co-morbidities prior to transplant } \\
\hline & Renal insufficiency $(\mathrm{Cr}>2)$ & 2.6 \\
\hline & Dialysis & 1.7 \\
\hline & Diabetes & 0.4 \\
\hline & Transfusion (between listing and transplant) & 31.9 \\
\hline & \multicolumn{2}{|l|}{ PRA prior to transplant } \\
\hline & Recent PRA $>10 \%$ & 23.4 \\
\hline & Peak PRA >10\% & 31.0 \\
\hline \multirow[t]{6}{*}{ Transplant } & Ischemic time (>4 h) & 31.5 \\
\hline & Ischemic time (mean \pm s.d.) & $3.5 \pm 1.3 \mathrm{~h}$ \\
\hline & Early rejection (within $1 \mathrm{yr}$ ) & 24.4 \\
\hline & Late rejection (after 1 yr) & 17.6 \\
\hline & Pacemaker implantation before discharge & 1.0 \\
\hline & Dialysis before discharge & 2.9 \\
\hline
\end{tabular}


Kobayashi et al.

Table 2. Donor characteristics $(\mathrm{N}=5211)$

\begin{tabular}{lll}
\hline Group & Characteristics & Percent \\
\hline Donor & Age (yr) & \\
$<1$ & 21.3 \\
$1-4$ & 24.6 \\
$5-9$ & 15.8 \\
$10-18$ & 20.5 \\
$>18$ & 17.8 \\
Age (mean \pm s.d.) & $9.3 \pm 10.3 \mathrm{yr}$ \\
Race & \\
Black & 17.1 \\
White & 62.9 \\
Others & 20.0 \\
Gender (female) & 42.3 \\
Co-morbidities & \\
Renal insufficiency (Cr $>2)$ & 4.8 \\
Diabetes & 0.8 \\
Hypertension & 1.3 \\
Cigarette use & 4.5 \\
Alcohol use & 2.5 \\
\hline
\end{tabular}

Table 3 shows the donor-recipient mismatch profile. The most common mismatch was overall HLA locus $(60.5 \%)$ and HLA DR locus $(55.5 \%)$, followed by race $(52.0 \%)$, gender $(49.0 \%)$, age $(36.1 \%)$, and $\mathrm{ABO}$ incompatible $(1.3 \%)$.

\section{Predictors of CAV}

The estimated incidence of CAV by the KaplanMeier method was $13 \%$ at five yr, $25 \%$ at $10 \mathrm{yr}$, and $54 \%$ at $15 \mathrm{yr}$ after transplant, respectively (Fig. 1).

Univariate analysis identified 16 factors associated with shorter CAV-free survival (Table 4). These factors included recipient age 1-4, 5-9, and 10-18 yr, donor age 1-4, 5-9, 10-18, and $>18 \mathrm{yr}$, re-transplant, recipient black race, recent and peak PRA $>10 \%$, recipient diabetes prior to transplant, donor alcohol use, donor cigarette use, early rejection, and transplant year after 2000. The Kaplan-Meier curves comparing CAV-free survival between the four age groups of recipients are shown in Fig. 2. No mismatch (age, gender, race, ABO, and HLA) is associated

Table 3. Mismatch characteristics between recipients and donors $(N=5211)$

\begin{tabular}{|c|c|c|}
\hline Mismatch & $\begin{array}{l}\text { Age mismatch } \\
\text { Gender mismatch } \\
\text { Race mismatch } \\
\text { ABO blood type } \\
\text { Identical } \\
\text { Compatible } \\
\text { Incompatible } \\
\text { HLA locus mismatch ( } \geq 5 \text { loci) } \\
\text { DR locus mismatch (2 loci) }\end{array}$ & $\begin{array}{r}36.1 \% \\
49.0 \% \\
52.0 \% \\
\\
77.6 \% \\
21.1 \% \\
1.3 \% \\
60.5 \% \\
55.5 \%\end{array}$ \\
\hline
\end{tabular}

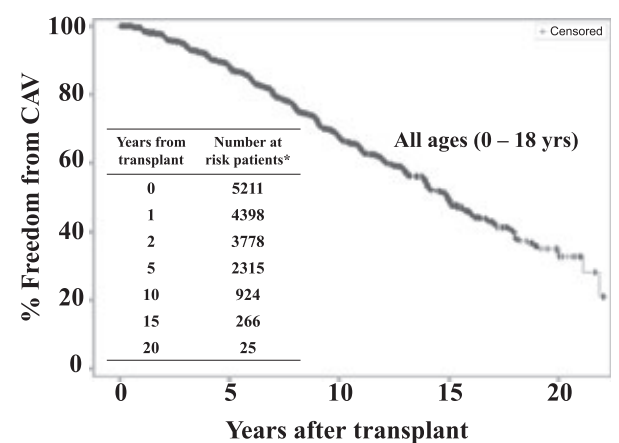

Fig. 1. Freedom from CAV using Kaplan-Meier survival analysis in all patients.

Table 4. Univariate analysis of CAV risk factors

\begin{tabular}{lllr}
\hline Variable & HR & $95 \% \mathrm{Cl}$ & p-Value \\
\hline Recipient age (reference: $<1$ yr) & & & \\
$\quad 1-4$ yr & 1.32 & $1.10-1.59$ & 0.0031 \\
$5-9$ yr & 1.65 & $1.34-2.01$ & $<0.0001$ \\
$\quad 10-18$ yr & 2.29 & $1.97-2.67$ & $<0.0001$ \\
Donor age (reference: $<1$ yr) & & & \\
$1-4$ yr & 1.43 & $1.17-1.73$ & 0.0004 \\
$5-9$ yr & 1.44 & $1.16-1.78$ & 0.0008 \\
$10-18$ yr & 2.28 & $1.88-2.77$ & $<0.0001$ \\
$>18$ yr & 3.14 & $2.57-3.83$ & $<0.0001$ \\
Diagnosis - re-transplant & 3.22 & $2.10-4.93$ & $<0.0001$ \\
Recipient race - black & 1.70 & $1.44-1.93$ & $<0.0001$ \\
Recent PRA $>10 \%$ & 1.20 & $1.03-1.39$ & 0.0194 \\
Peak PRA $>10 \%$ & 1.27 & $1.10-1.47$ & 0.0012 \\
Recipient diabetes & 3.42 & $1.63-7.21$ & 0.0012 \\
Donor alcohol use & 2.52 & $1.88-3.38$ & $<0.0001$ \\
Donor cigarette use & 2.53 & $2.03-3.15$ & $<0.0001$ \\
Early rejection $(<1$ yr) & 1.69 & $1.47-1.93$ & $<0.0001$ \\
Transplant year after 2000 & 1.31 & $1.14-1.51$ & $<0.0001$ \\
\hline
\end{tabular}

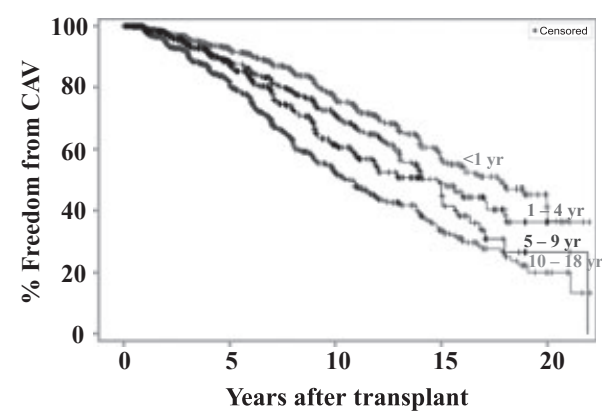

Fig. 2. Freedom from CAV using Kaplan-Meier survival analysis in patients stratified by age group (age $<1,1-4,5-9$, and $10-18 \mathrm{yr}$ ).

with shorter CAV-free survival in univariate analysis.

Multivariate analysis was performed on all variables associated with shorter CAV-free survival in univariate analysis. Factors significantly associated with shorter CAV-free survival in the final model (Table 5) were recipient age 1-4 yr 
$(\mathrm{HR}=1.25, \mathrm{p}=0.0167)$, recipient age 5-9 $\mathrm{yr}$ $(\mathrm{HR}=1.45, \mathrm{p}=0.0004)$, recipient age $10-$ $18 \mathrm{yr} \quad(\mathrm{HR}=1.83, \mathrm{p}<0.0001)$, donor age $>18$ yr $(\mathrm{HR}=1.34, \mathrm{p}=0.0256)$, re-transplantation $(\mathrm{HR}=2.14, \mathrm{p}<0.0001)$, recipient black race $(\mathrm{HR}=1.55, \mathrm{p}<0.0001)$, and donor cigarette use $(H R=1.54, \mathrm{p}=0.0001)$.

Impact of coronary allograft vasculopathy on graft survival

Median patient and graft survivals were 17.5 and $12.8 \mathrm{yr}$, respectively. Median graft survivals for patients with and without CAV were 10.7 and $14.8 \mathrm{yr}(\mathrm{p}<0.001)$, respectively. The time from development of CAV to graft loss (defined as death or re-transplantation) was analyzed by the Kaplan-Meier survival method (Fig. 3). The estimated graft survival after CAV development was $42 \%$ at five yr, $24 \%$ at $10 \mathrm{yr}$, and $16 \%$ at $15 \mathrm{yr}$. There was an initial drop off at time 0 , indicating that approximately $23.5 \%$ of the patients had CAV diagnosed and graft failure simultaneously.

\section{Discussion}

To our knowledge, this is the largest study describing predictors of CAV development in pediatric heart transplant recipients. Our final

Table 5. Multivariate analysis of CAV risk factors

\begin{tabular}{lrrr}
\hline Variable & HR & $95 \% \mathrm{Cl}$ & p-Value \\
\hline Recipient age (reference: $<1$ yr) & & & \\
$\quad 1-4$ yr & 1.25 & $1.04-1.51$ & 0.0167 \\
$5-9$ yr & 1.45 & $1.18-1.77$ & 0.0004 \\
$10-18$ yr & 1.83 & $1.54-2.18$ & $<0.0001$ \\
Donor age $>18$ yr (reference: $<18$ yr) & 1.34 & $1.11-1.60$ & 0.0256 \\
Diagnosis - re-transplant & 2.14 & $1.70-2.70$ & $<0.0001$ \\
Recipient race - black & 1.55 & $1.33-1.80$ & $<0.0001$ \\
Donor cigarette use & 1.54 & $1.20-1.98$ & 0.0001 \\
\hline
\end{tabular}

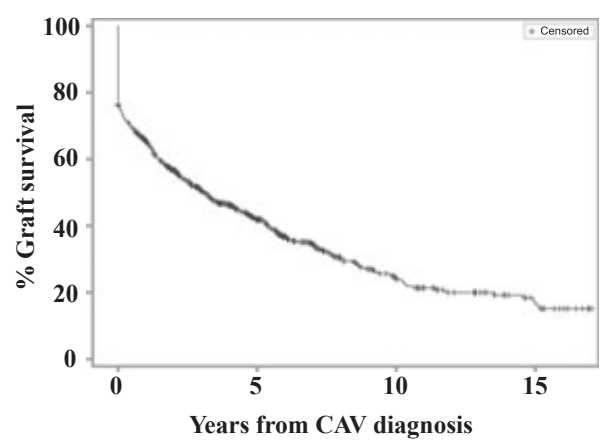

Fig. 3. Graft survival after diagnosis of CAV by the Kaplan-Meier survival method in all patients. *Number of patients who were still CAV-free minus those that are censored out. multivariate model showed that older recipient age, donor age $(>18 \mathrm{yr})$, recipient black race, re-transplantation, and donor cigarette use were highly associated with shorter CAV-free survival in pediatric heart transplant recipients. In univariate analysis, donor age $<1$ yr was associated with the lowest risk of CAV but became insignificant in the final model. This was most likely because donor age was highly correlated with recipient age. Transplant after year 2000 was associated with shorter CAV-free survival in univariate analysis. However, this era effect became insignificant in multivariate analysis, most likely because more high-risk heart transplants were performed after year 2000. Degree of HLA, ABO, age, gender, and race mismatch were not significant predictors of CAV. PRA $>10 \%$, recipient diabetes status, donor alcohol use, and early rejection were significant in univariate analysis but were not in multivariate analysis. Although cigarette use is likely associated with older donor age $(>18 \mathrm{yr})$, donor cigarette use remained significant in our final multivariate model even after adjustment for age. Donor cigarette use may represent other unmeasured risk for donor pre-existing coronary artery disease.

Two previous studies describe risk factors for CAV in pediatric heart transplant recipients. The PHTS report analyzed data from 751 children who had coronary angiograms after heart transplant (3). The incidence of angiographic coronary abnormalities at one, three, and five yr was $2 \%, 9 \%$, and $17 \%$, respectively. Risk factors for CAV were recipient age $\geq 12 \mathrm{yr}$, donor age $>30 \mathrm{yr}$, and early rejection ( $\geq 2$ times within one yr of transplant). In a more recent report from the ISHLT (2), 34\% of patients were estimated to develop CAV by $10 \mathrm{yr}$ after transplant. Freedom from CAV 10 yr after transplant was different among recipient age groups: $73 \%$ for age $<1 \mathrm{yr}, 73 \%$ for $1-10 \mathrm{yr}$, and $53 \%$ for 11-18 yr. Our estimated incidence of CAV was consistent with previous data. Graft failure after CAV development was high, confirming that CAV development affected eventual graft survival. In our cohort, patients with CAV had a $4.1 \mathrm{yr}$ shorter median graft survival than those without CAV. We again demonstrated that recipient age, donor age, and re-transplantation were associated with shorter CAV-free survival. A new finding of our study was the significant association between recipient black race and shorter $\mathrm{CAV}$-free survival. Black recipients are known to have increased risk of long-term graft loss compared with other races (5). Biological and socioeconomic factors may also contribute to a higher 
risk of rejection, resulting in worse post-transplant graft survival in this group (6).

One of our study questions was to examine the association between HLA mismatch and CAV. HLA mismatch can result in development of DSA, which can contribute to the development of CAV by antibody-mediated rejection. CAVfree survival was not associated with overall HLA mismatch level or HLA DR mismatch at the time of transplant. The presence of DSA is not available in the OPTN/UNOS database.

Traditional and non-traditional risk factors for CAV have been described primarily in adults (7). Traditional risk factors include hyperlipidemia, diabetes, and hypertension; non-traditional risk factors include cytomegalovirus infection, HLA mismatch, antibody-mediated rejection, and mode of donor brain death. Because graft survival is $48 \%$ at five $\mathrm{yr}$ after the diagnosis of CAV (2), the prevention of CAV is critical to improved graft survival. Transplant recipients with known risk factors for CAV should be monitored closely and receive early intervention to reduce $\mathrm{CAV}$ risk.

\section{Limitation}

The advantage of our study is that submission of data to the OPTN/UNOS database is mandatory for transplant registrants and recipients, thus increasing the statistical power to identify risk factors for rare events such as CAV. The OPTN/ UNOS database is not structured specifically to be a research database and thus has inherent limitations. There was no uniform definition or monitoring for CAV, and no CAV severity given. Accordingly, there may be potential misclassification bias. The incidence of CAV is dependent to some extent on the surveillance strategy of individual center. Some putative CAV variables such as hypertension, cytomegalovirus infection, Epstein-Barr virus infection, dyslipidemia, and crossmatch at transplant as well as antilipid and antihypertensive medications were not included in our analysis because these data were not routinely available for all patients in the database. In terms of acute rejection, antibody-mediated and cellular rejection was not separately recorded, and no variables describing the frequency or severity of acute rejection episodes were available in the database. Because of the wide variety and change of immunosuppressive agents and regimen over the post-transplant course, the effect of each agent and regimen on CAV development could not be accurately assessed in our model.

\section{Conclusions}

Older recipient and donor age, recipient black race, donor cigarette use, and re-transplantation were highly associated with shorter time from transplantation to development of CAV in pediatric heart transplant recipients. The identification of higher risk recipients may urge transplant providers to more closely monitor these patients in terms of other modifiable traditional risk factors, thus potentially reducing incidence/severity of $\mathrm{CAV}$, and increasing graft survival.

\section{Authors' contributions}

D. Kobayashi participated in study design, development of question, and drafting of this manuscript. W. Du participated in data analysis and critical writing, revision, and approval of this manuscript. T. L'Ecuyer participated in study design, development of question, revision, and approval of this manuscript.

\section{References}

1. Schumacher KR, Gajarski RJ, Urschel S. Pediatric coronary allograft vasculopathy - a review of pathogenesis and risk factors. Congenit Heart Dis 2012: 7: 312-323.

2. Kirk R, Edwards LB, Kucheryavaya AY, et al. The Registry of the International Society for Heart and Lung Transplantation: Thirteenth official pediatric heart transplantation report2010. J Heart Lung Transplant 2010: 29: 1119-1128.

3. Pahl E, Naftel DC, Kuhn MA, et al. The impact and outcome of transplant coronary artery disease in a pediatric population: A 9-year multi-institutional study. J Heart Lung Transplant 2005: 24: 645-651.

4. United Network of Organ Sharing (UNOS) website. Available at: http://www.unos.org/ (accessed March 20, 2012)

5. Mahle WT, Kanter KR, Vincent RN. Disparities in outcome for black patients after pediatric heart transplantation. J Pediatr 2005: 147: 739-743.

6. Singh TP, Almond CS, Gauvreau K. Improved survival in pediatric heart transplant recipients: Have white, black and Hispanic children benefited equally? Am J Transplant 2011: 11: 120-128.

7. Colvin-Adams M, Agnihotri A. Cardiac allograft vasculopathy: Current knowledge and future direction. Clin Transplant 2011: 25: 175-184. 